\title{
Padrões de identidade e qualidade de fermentados acéticos comerciais de frutas e vegetais
}

\author{
Quality pattern and identity of commercial fruit and vegetable vinegar (Acetic acid fermentation)
}

\author{
Fabíola Pedrosa Peixoto MARQUES ${ }^{1 \star}$, Wilma SPINOSA², Kátia Flávia FERNANDES ${ }^{3}$, \\ Carlos Frederico de Souza CASTRO $^{4}$, Márcio CALIARI ${ }^{5}$
}

\begin{abstract}
Resumo
Este trabalho objetivou identificar padrões de identidade e qualidade de vinagres e quantificar a presença de compostos fenólicos totais em vinagres produzidos a partir de frutas e vegetais. Analisaram-se, em triplicata, vinagres de cana-de-açúcar, cana-de-açúcar com milho, kiwi, laranja, laranja com mel, maçã, arroz, manga, maracujá, milho, tangerina, tangerina com milho, vinagre de vinho tinto e vinagre de vinho branco. Foram realizadas análises de extrato seco, densidade, acidez volátil, $\mathrm{pH}$, cinzas, grau alcoólico, açúcares redutores e polifenóis totais. Os resultados foram submetidos à ANOVA e as médias destes ao teste de Tukey ao nível de $5 \%$. Houve correlação estatística entre extrato seco e densidade. Todas as amostras apresentaram valores de $0 \%$ de álcool. A presença do mel na formulação do vinagre de laranja com mel $\left(43,27 \mathrm{mg}\right.$ EAG $\left.100 . \mathrm{mL}^{-1}\right)$ contribuiu para o aumento do conteúdo de polifenóis totais em relação ao de laranja $\left(28,83 \mathrm{mg}\right.$ EAG.100 mL $\left.\mathrm{m}^{-1}\right)$ em 50\%. O fermentado de laranja com mel apresentou teor de polifenóis totais (43,27 mg EAG.100 $\left.\mathrm{mL}^{-1}\right)$ maior que o vinagre de vinho tinto (31,86 mg EAG.100 mL $\mathrm{m}^{-1}$ ), assim como os de laranja e tangerina (34,36 mg EAG.100 $\left.\mathrm{mL}^{-1}\right)$. Conclui-se que houve diferenças entre as amostras para os parâmetros analisados e que os fermentados acéticos de laranja com mel, laranja e tangerina apresentam maior conteúdo de polifenóis totais que o de vinho tinto.
\end{abstract}

Palavras-chave: fermentado acético; polifenóis totais; antioxidantes.

\begin{abstract}
This work aimed to identify the patterns of identity and quality of vinegars and quantify the presence of total phenolics compounds in fruit and vegetable vinegars, Vinegars made from sugar cane, sugar cane and corn, kiwi, orange, orange and honey, apple, rice, mango, passion fruit, corn, tangerine, tangerine and corn, red wine, and white wine were analyzed in triplicates. Analyses of dry extract, density, volatile acidity, $\mathrm{pH}$, ashes, alcoholic content, reducing sugars, and total polyphenols were performed. The results were submitted to ANOVA followed by the Tukey's test at $5 \%$ significance. There was a statistical correlation between the dry extract and density. All samples presented values of $0 \%$ of alcohol. The presence of honey in the vinegar formulation made from orange and honey (43.27 mg EAG.100 $\left.\mathrm{mL}^{-1}\right)$ contributed to the increase of total polyphenols content by $50 \%$ in relation to the vinegar made from orange ( $28.83 \mathrm{mg}$ EAG.100 $\left.\mathrm{mL}^{-1}\right)$. The orange and honey vinegar presented total polyphenols content (43.27 mg EAG.100 $\left.\mathrm{mL}^{-1}\right)$ higher than that of the red wine vinegar $\left(31.86 \mathrm{mg} \mathrm{EAG.100} \mathrm{mL}^{-1}\right)$, the orange vinegar, and the tangerine vinegar ( $34.36 \mathrm{mg}$ EAG. $\left.100 \mathrm{~mL}^{-1}\right)$. It can be concluded that there were differences among the samples and the analyzed parameters, and that the vinegars made from orange and honey, orange, and tangerine presented higher total polyphenols contents than those of the red wine vinegar.
\end{abstract}

Keywords: fermented acetic; total polyphenols; antioxidants.

\section{Introdução}

O fermentado acético é definido como o produto obtido da fermentação acética do fermentado alcoólico de mosto de frutas, cereais ou de outros vegetais, de mel, ou da mistura de vegetais, ou ainda de mistura hidroalcoólica. Deve apresentar acidez volátil mínima de $4 \mathrm{~g} .100 \mathrm{~mL}^{-1}$, expressa em ácido acético, podendo ser acrescido de vegetais, partes de vegetais ou extratos vegetais aromáticos ou de sucos, aromas naturais ou condimentos. O fermentado acético pode apresentar várias classificações, de acordo com a origem da matéria-prima, sendo designados de fermentados acéticos ou vinagres, seguidos do nome da matéria-prima de origem (BRASIL, 1999).
A produção do vinagre ocorre por dois processos bioquímicos distintos resultantes da ação de microrganismos através da fermentação alcoólica pela ação de leveduras, usualmente espécies de Saccharomyces, sobre as matérias-primas açucaradas e/ou amiláceas, seguida de fermentação acética, pela ação de bactérias aeróbias do gênero Acetobacter (BORTOLINI; SANT'ANNA; TORRES, 2001; GRANANDA et al., 2000; TESFAYE et al., 2002).

A utilização do vinagre na alimentação pode ocorrer nas formas de condimento, conferindo sabor ácido, como

\footnotetext{
Recebido para publicação em 27/3/2008

Aceito para publicação em 4/1/2009 (003370)

${ }^{1}$ Ciência e Tecnologia de Alimentos, Universidade Federal de Goiás - UFG, Goiânia, GO, Brasil, E-mail:fabiolappm@hotmail.com

2 Indústria de Vinagres Dom Spinosa, Assis, SP, Brasil

${ }^{3}$ Instituto de Ciências Biológicas - ICB, Universidade Federal de Goiás - UFG, Goiânia, GO, Brasil

${ }^{4}$ Instituto Federal de Educação Tecnológica - IFET, Rio Verde, GO, Brasil

${ }_{5}^{5}$ Escola de Agronomia e Engenharia de Alimentos, Universidade Federal de Goiás - UFG, Goiânia, GO, Brasil

${ }^{*}$ A quem a correspondência deve ser enviada
} 
conservante evitando o crescimento de microrganismos (ENTANI et al., 1998; SEGUN; KARAPINAR, 2004), além de apresentar bastante utilidade como amaciante de carnes temperadas e legumes em conservas (BORTOLINI; SANT 'ANNA; TORRES, 2001; GRANANDA et al., 2000). Além disso, o vinagre pode apresentar efeitos fisiológicos positivos em relação à regulação da glicose sanguínea (EBIHARA; NAKAJIMA, 1988; JOHNSTON; BULLER, 2005), controle da pressão arterial, auxílio da digestão, estimulação do apetite (XU; TAO; AO, 2007) e promoção da absorção de cálcio (HADFIELD; BEARD; LEONARD-GREEN, 1989; XU; TAO; AO, 2007).

A fabricação de vinagre proporciona um meio de utilização de matéria-prima inaproveitável dos estabelecimentos industriais (MALDONADO; ROLZ; CABRERA, 1975) de frutas e especialmente de propriedades rurais que não poderiam competir no mercado (EVANGELISTA, 2001) constituindo, então, uma forma de aproveitamento de excedentes de safra (BROTOLINI; SANT'ANNA; TORRES, 2001; OLIVEIRA et al., 1987). É válido ressaltar que os vinagres de frutas são considerados superiores em qualidades sensoriais e nutritivas, quando comparados a outros tipos de vinagres, além de apresentarem vitaminas, ácidos orgânicos, proteínas e aminoácidos provenientes do fruto e da fermentação alcoólica (AQUARONE; ZANCANARO Jr., 1990). Porém, a qualidade final do vinagre pode ser influenciada pela matéria-prima utilizada como substrato para sua fabricação, pelo sistema de acetificação utilizado e pela presença ou não de processos de envelhecimento em madeira (TESFAYE et al., 2002).

Os polifenóis totais são compostos que apresentam atividade antirradical livre (REYNERTSON et al., 2006; KUSKOSKI et al., 2006), encontrados em diferentes alimentos como vegetais, frutas, chocolate, chá, café, vinho, sucos de uva e vinagre, em diferentes concentrações (DÁVALOS; BARTOLOMÉ; GÓMEZ-CORDOVÉS, 2005). Os polifenóis totais têm sido estudados devido à sua influência na qualidade dos alimentos, englobando uma variedade significativa de substâncias que apresentam atividades farmacológicas, inibição da oxidação lipídica, proliferação de fungos, além de participarem em processos responsáveis pela cor, adstringência e aroma em vários alimentos (SOARES, 2002).

Observa-se a presença de poucos estudos avaliando a capacidade antioxidante de vinagres (NISHIDAI et al., 2000), ressaltando-se que a composição química deste está relacionada com a qualidade, a tecnologia e a matéria-prima utilizada na sua elaboração (RIZZON; MIELE, 1998). Como poucos trabalhos abordam o vinagre sob o ponto de vista nutricional, o presente trabalho objetivou identificar alguns padrões de identidade e qualidade de vinagres, bem como quantificar a presença de compostos fenólicos totais, como parâmetros nutricionais importantes em vinagres produzidos a partir de frutas e vegetais, comparando-os com vinagres de vinho.

\section{Material e métodos}

Foram analisadas doze (12) amostras de vinagres comerciais de frutas e vegetais produzidas e comercializadas por uma indústria vinagreira localizada na cidade de Assis - SP. Além disso, foram analisadas também duas (2) amostras de vinagres de vinho (branco e tinto), a título de comparação com os vinagres de frutas e vegetais. Os vinagres foram adquiridos aleatoriamente no local onde eram comercializados e uma amostra de cada tipo foi analisada.

Analisaram-se, em três repetições, as amostras de vinagres de cana-de-açúcar (VC); vinagre de cana-de-açúcar com milho (VCMi); vinagre de kiwi (VK); vinagre de laranja (VL); vinagre de laranja com mel (VLMl); vinagre de maçã (VMç); vinagre de arroz (Var); vinagre de manga (VMg); vinagre de maracujá (VMr); vinagre de milho (VMi); vinagre de tangerina (VTg); vinagre de tangerina com milho (VTgMi); vinagre de vinho tinto (VVT); e vinagre de vinho branco (VVB).

As amostras foram submetidas a análises físico-químicas de extrato seco total, densidade relativa a $20^{\circ} \mathrm{C}$, acidez volátil, $\mathrm{pH}$, cinzas, grau alcoólico real, além de quantificação de açúcares redutores e polifenóis totais. As análises físico-químicas foram realizadas no Laboratório de Físico-Química da Faculdade de Agronomia e Engenharia de Alimentos da Universidade Federal de Goiás - UFG, segundo metodologias propostas por Brasil (2005). Buscando-se utilizar uma determinação ainda pouco explorada em vinagres, a quantificação de açúcares redutores foi realizada segundo Miller (1959), com adaptações, utilizando o ácido 3-5-dinitrossalicílico (ADNS). As análises de polifenóis totais foram determinados e Ciocalteau (1957 apud SOUSA et al., 2007), com adaptações, respectivamente.

Os resultados analíticos obtidos foram submetidos à análise de variância univariada (ANOVA) e as médias determinadas para cada experimento ao teste de Tukey com $5 \%$ de probabilidade de erro, utilizando-se o aplicativo SAS for Windows, versão 6.12 (SAS, 1996).

\section{Resultados e discussão}

Os resultados das análises físico-químicas realizadas estão apresentados na Tabela 1 .

De acordo com análise da Tabela 1 observa-se que para todas as variáveis estudadas - extrato seco total, densidade relativa a $20^{\circ} \mathrm{C}$, acidez volátil, $\mathrm{pH}$, cinzas, açúcares redutores e polifenóis totais - houve a formação de grupos, o que indica que as amostras são heterogêneas com base no teste de Tukey a $5 \%$ de significância.

\subsection{Padrões de identidade e qualidade}

\section{Extrato seco total}

O conteúdo de extrato seco total representa o material mineral e orgânico resultante da evaporação da água e substâncias voláteis da amostra. Observa-se que, em relação ao teor de extrato seco total, os vinagres de manga (VMg), arroz (Var) e kiwi (VK) não diferiram significativamente entre si ( $\mathrm{p}>0,05)$, com valores em torno de 14,1 a 14,8 g. $\mathrm{L}^{-1}$. Os vinagres de laranja com mel (VLML) e laranja (VL) apresentaram os maiores valores de extrato seco, 48,8 a 38,3 g.L $\mathrm{L}^{-1}$, respectivamente. Além do vinagre de laranja (VL) ter apresentado teor elevado de extrato seco, a presença do mel no fermentado acético à base 
Tabela 1. Resultados obtidos para extrato seco, densidade relativa a $20^{\circ} \mathrm{C}$, acidez volátil, $\mathrm{pH}$, cinzas, açúcares redutores e polifenóis totais (média, desvio padrão e coeficiente de determinação do erro analítico) das amostras de vinagres analisadas.

\begin{tabular}{|c|c|c|c|c|c|c|c|}
\hline Vinagres & ES $\left(\right.$ g. $\left.L^{-1}\right)$ & Dens (g.mL $\left.{ }^{-1}\right)$ & $\mathrm{AV}(\%)$ & $\mathrm{pH}$ & $\operatorname{Cin}\left(\right.$ g. $\left.\mathrm{L}^{-1}\right)$ & AR $\left(\right.$ g..$\left.L^{-1}\right)$ & PT $(\mathrm{mg} \text { EAG } 100 \mathrm{~mL})^{-1}$ \\
\hline VLMl & $\begin{array}{c}48,8 \mathrm{a} \pm 0,683 \\
(1,398)\end{array}$ & $\begin{array}{c}1,0206 \mathrm{a} \pm 0,001 \\
(0,065)\end{array}$ & $\begin{array}{c}2,53 \mathrm{~h} \pm 0,000 \\
(0,000)\end{array}$ & $\begin{array}{c}3,74 \mathrm{a} \pm 0,046 \\
(1,225)\end{array}$ & $\begin{array}{c}5,14 \mathrm{a} \pm 0,251 \\
(4,876)\end{array}$ & $\begin{array}{c}4,35 \text { e } \pm 0,052 \\
(1,195)\end{array}$ & $\begin{array}{c}43,27 \mathrm{a} \pm 0,301 \\
(0,696)\end{array}$ \\
\hline VL & $\begin{array}{c}38,3 \mathrm{~b} \pm 0,675 \\
(1,761)\end{array}$ & $\begin{array}{c}1,0198 \mathrm{a} \pm 0,000 \\
(0,032)\end{array}$ & $\begin{array}{l}2,74 \mathrm{gh} \pm 0,070 \\
(2,564)\end{array}$ & $\begin{array}{c}3,40 \mathrm{c} \pm 0,025 \\
(0,739)\end{array}$ & $\begin{array}{l}3,65 \mathrm{bc} \pm 0,008 \\
(0,219)\end{array}$ & $\begin{array}{l}4,12 \text { e } \pm 0,060 \\
(1,462)\end{array}$ & $\begin{array}{l}28,83 \mathrm{~d} \pm 0,397 \\
(1,377)\end{array}$ \\
\hline VTgMi & $\begin{array}{c}23,4 c \pm 0,427 \\
(1,823)\end{array}$ & $\begin{array}{c}1,0124 \mathrm{c} \pm 0,000 \\
(0,029)\end{array}$ & $\begin{array}{l}3,47 \text { cdef } \pm 0,081 \\
(2,341)\end{array}$ & $\begin{array}{c}3,54 \mathrm{~b} \pm 0,044 \\
(1,231)\end{array}$ & $\begin{array}{c}3,46 c \pm 0,018 \\
(0,529)\end{array}$ & $\begin{array}{l}2,53 \mathrm{~g} \pm 0,095 \\
(3,726)\end{array}$ & $\begin{array}{l}27,61 \mathrm{~d} \pm 0,284 \\
(1,030)\end{array}$ \\
\hline $\mathrm{VMg}$ & $\begin{array}{c}14,8 \text { e } \pm 0,302 \\
(2,028)\end{array}$ & $\begin{array}{c}1,0123 \mathrm{c} \pm 0,000 \\
(0,025)\end{array}$ & $\begin{array}{c}3,28 \operatorname{defg} \pm 0,215 \\
(6,547)\end{array}$ & $\begin{array}{c}2,65 \mathrm{~h} \pm 0,015 \\
(0,576)\end{array}$ & $\begin{array}{c}2,25 \text { e } \pm 0,073 \\
(3,227)\end{array}$ & $\begin{array}{c}8,74 \mathrm{a} \pm 0,000 \\
(0,000)\end{array}$ & $\begin{array}{c}16,80 \mathrm{f} \pm 0,734 \\
(4,371)\end{array}$ \\
\hline Var & $\begin{array}{c}14,3 \text { e } \pm 0,378 \\
(2,638)\end{array}$ & $\begin{array}{l}1,0124 \mathrm{c} \pm 0,000 \\
(0,020)\end{array}$ & $\begin{array}{c}3,70 \text { bcde } \pm 0,325 \\
(8,770)\end{array}$ & $\begin{array}{c}2,89 \mathrm{fg} \pm 0,017 \\
(0,599)\end{array}$ & $\begin{array}{c}3,75 \mathrm{~b} \pm 0,115 \\
(3,061)\end{array}$ & $\begin{array}{c}6,65 \mathrm{~b} \pm 0,117 \\
(1,760)\end{array}$ & $\begin{array}{l}6,54 \mathrm{~h} \pm 0,050 \\
(0,765)\end{array}$ \\
\hline VK & $\begin{array}{c}14,1 \mathrm{e} \pm 0,298 \\
(2,100)\end{array}$ & $\begin{array}{c}1,0113 \mathrm{~cd} \pm 0,001 \\
(0,053)\end{array}$ & $\begin{array}{c}3,10 \mathrm{fg} \pm 0,070 \\
(2,273)\end{array}$ & $\begin{array}{c}2,97 \text { ef } \pm 0,006 \\
(0,194)\end{array}$ & $\begin{array}{c}1,66 \mathrm{f} \pm 0,019 \\
(1,137)\end{array}$ & $\begin{array}{c}5,12 \mathrm{~d} \pm 0,147 \\
(2,877)\end{array}$ & $\begin{array}{c}17,61 \mathrm{f} \pm 0,055 \\
(0,313)\end{array}$ \\
\hline $\mathrm{VMr}$ & $\begin{array}{l}9,3 \mathrm{~g} \pm 0,222 \\
(2,378)\end{array}$ & $\begin{array}{c}1,0089 \text { ef } \pm 0,000 \\
(0,026)\end{array}$ & $\begin{array}{c}3,91 \mathrm{bc} \pm 0,107 \\
(2,744)\end{array}$ & $\begin{array}{l}3,33 c \pm 0,006 \\
(0,173)\end{array}$ & $\begin{array}{c}1,45 \mathrm{f} \pm 0,024 \\
(1,653)\end{array}$ & $\begin{array}{c}1,15 \mathrm{i} \pm 0,010 \\
(0,870)\end{array}$ & $\begin{array}{l}9,79 \mathrm{~g} \pm 0,247 \\
(2,518)\end{array}$ \\
\hline VMç & $\begin{array}{c}8,9 \text { gh } \pm 0,311 \\
(3,468)\end{array}$ & $\begin{array}{c}1,0109 \mathrm{~d} \pm 0,000 \\
(0,019)\end{array}$ & $\begin{array}{c}4,17 \mathrm{~b} \pm 0,041 \\
(0,973)\end{array}$ & $\begin{array}{c}3,10 \mathrm{~d} \pm 0,017 \\
(0,559)\end{array}$ & $\begin{array}{l}1,49 \mathrm{f} \pm 0,012 \\
(0,821)\end{array}$ & $\begin{array}{c}6,22 c \pm 0,150 \\
(2,416)\end{array}$ & $\begin{array}{c}22,61 \mathrm{e} \pm 0,218 \\
(0,964)\end{array}$ \\
\hline VVB & $\begin{array}{l}8,0 \mathrm{~h} \pm 0,224 \\
\quad(2,784)\end{array}$ & $\begin{array}{c}1,0151 \mathrm{~b} \pm 0,001 \\
(0,063)\end{array}$ & $\begin{array}{c}4,03 \mathrm{~b} \pm 0,494 \\
(12,251)\end{array}$ & $\begin{array}{l}2,88 \mathrm{~g} \pm 0,000 \\
(0,000)\end{array}$ & $\begin{array}{c}0,72 \mathrm{~h} \pm 0,009 \\
(1,281)\end{array}$ & $\begin{array}{c}1,12 \mathrm{i} \pm 0,012 \\
(1,028)\end{array}$ & $\begin{array}{c}9,53 \mathrm{~g} \pm 0,308 \\
(3,233)\end{array}$ \\
\hline VCMi & $\begin{array}{c}5,6 \mathrm{i} \pm 0,144 \\
(2,554)\end{array}$ & $\begin{array}{c}1,0079 \mathrm{fg} \pm 0,000 \\
(0,031)\end{array}$ & $\begin{array}{c}3,17 \text { efg } \pm 0,070 \\
(2,222)\end{array}$ & $\begin{array}{c}3,03 \mathrm{de} \pm 0,045 \\
\quad(1,485)\end{array}$ & $\begin{array}{c}0,99 \mathrm{~g} \pm 0,010 \\
(1,012)\end{array}$ & $\begin{array}{l}1,31 \text { hi } \pm 0,060 \\
\quad(4,580)\end{array}$ & $\begin{array}{c}5,13 \mathrm{~h} \pm 0,069 \\
(1,351)\end{array}$ \\
\hline VMi & $\begin{array}{c}5,3 \mathrm{i} \pm 0,272 \\
(5,155)\end{array}$ & $\begin{array}{c}1,0077 \mathrm{~g} \pm 0,001 \\
(0,059)\end{array}$ & $\begin{array}{c}3,75 \text { bcd } \pm 0,081 \\
(2,165)\end{array}$ & $\begin{array}{c}3,05 \mathrm{de} \pm 0,047 \\
(1,546)\end{array}$ & $\begin{array}{c}0,91 \mathrm{gh} \pm 0,018 \\
(0,867)\end{array}$ & $\begin{array}{c}1,13 \mathrm{i} \pm 0,006 \\
(0,509)\end{array}$ & $\begin{array}{c}3,13 \mathrm{i} \pm 0,000 \\
(0,000)\end{array}$ \\
\hline
\end{tabular}

${ }^{\star}$ Coeficiente de determinação geral; ${ }^{* *}$ coeficiente de determinação do erro analítico; letras iguais nas colunas - vinagres não diferiram entre si (P > 0,05) pelo teste de Tukey. ES (extrato seco); Dens (densidade relativa a $20^{\circ} \mathrm{C}$ ); AV (acidez volátil); Cin (cinzas); GA (grau alcoólico real); AR (açúcares redutores); e PT (polifenóis totais).

de laranja com mel (VLMl), possivelmente, contribuiu para o aumento no teor de extrato seco deste produto.

Houve diferença significativa $(\mathrm{p} \leq 0,05)$ entre os vinagres de vinho tinto $\left(10,5\right.$ g. $\left.\mathrm{L}^{-1}\right)$ e vinho branco $\left(8,0\right.$ g. $\left.\mathrm{L}^{-1}\right)$. Em estudos realizados por Rizzon e Miele (1998) referentes às características analíticas de vinagres comerciais de vinhos brasileiros, os autores encontraram uma faixa média no conteúdo de extrato seco de 9,78 g.L. $\mathrm{L}^{-1}$ para vinagres de vinho tinto e $9,32 \mathrm{~g} . \mathrm{L}^{-1}$ para vinagres de vinho branco, valores aproximadamente $7 \%$ menor e $16 \%$ maior, respectivamente, que dos VVT e VVB analisados no presente trabalho.

É válido ressaltar a presença de valores superiores do teor de extrato seco dos vinagres de manga (VMg), arroz (Var), kiwi (VK), laranja (VL) e laranja com mel (VLMl) em relação aos vinagres convencionais de vinho tinto (VVT, 10,5 g. $\mathrm{L}^{-1}$ ) e vinho branco (VVB, 8,0 g.L $\mathrm{L}^{-1}$ ). Acredita-se que as matérias-primas utilizadas, bem como os métodos de filtração e envelhecimento dos produtos durante a fabricação, podem ter interferido nos resultados superiores de extrato seco. Além disso, há de se considerar que a estocagem do vinagre por determinado período de tempo em madeira pode produzir modificações no seu conteúdo de sólidos (WHITE, 1971), embora não tenha sido mencionada nas embalagens dos produtos a presença de nenhuma forma de envelhecimento. A qualidade da filtração do vinagre após sua produção pode influenciar diretamente na quantidade de sólidos (orgânicos e inorgânicos) finais no vinagre. No processo industrial mais comum de produção de vinagre, o submerso, há produção de vinagre relativamente turvo, que pode conter em suspensão bactérias acéticas e substâncias sólidas originadas da matéria-prima. Este, porém necessita ser acrescido intimamente de um agente clarificante (geralmente a bentonita), deixado em repouso para sedimentação e, posteriormente, filtrado (em filtro-prensa) (PALMA; CARVALHO; GAVÓGLIO, 2001). Uma não observância na qualidade deste processo pode resultar em vinagres com teores sólidos finais mais altos.

Os menores valores para esta determinação foram apresentados pelos vinagres de cana-de-açúcar com milho (VCMi, 5,6 g.L. $\mathrm{L}^{-1}$ ) e milho (VMi 5,3 g.. $\mathrm{L}^{-1}$ ), que não diferiram 
significativamente entre si $(\mathrm{p}>0,05)$, assim como vinagres de cana-de-açúcar (VC, 10,6 g.L L $^{-1}$ ) e vinho tinto (VVT, 10,5 g.L $\left.\mathrm{L}^{-1}\right)$.

\section{Densidade relativa a $20^{\circ} \mathrm{C}$}

A densidade relativa a $20^{\circ} \mathrm{C}$ baseia-se na relação existente entre o peso específico da amostra a $20^{\circ} \mathrm{C}$ em relação ao peso específico da água a $20^{\circ} \mathrm{C}$ que, nas mesmas condições, é igual a 1,0 (um). Observou-se que não houve diferença significativa ( $p>0,05)$ entre os vinagres de tangerina com milho (VTMi), manga (VMg), arroz (Var) e vinagre de vinho tinto (VVT), que variaram de 1,0122 a $1,0124 \mathrm{~g} \cdot \mathrm{mL}^{-1}$. Da mesma forma não se observou diferença significativa $(p>0,05)$ entre os vinagres de milho (VMi), 1,0077 g.mL $\mathrm{mL}^{-1}$ e cana-de-açúcar com milho (VCMi), 1,0079 g.mL $\mathrm{mL}^{-1}$.

Os VVT (1,0122 g.mL $\left.\mathrm{mL}^{-1}\right)$ e VVB (1,0151 g.mL $\left.\mathrm{mL}^{-1}\right)$ diferiram significativamente entre si $(\mathrm{p} \leq 0,05)$ quanto à densidade. Rizzon e Miele (1998) encontraram valores menores a partir da caracterização analítica de vinagres comerciais de vinhos brasileiros, sendo, para vinagre de vinho tinto, 1,0103 g. $\mathrm{mL}^{-1} \mathrm{e}$, para vinagre de vinho branco, 1,0009 g. $\mathrm{mL}^{-1}$; valores menores na ordem de 0,2 e 1,4\%, respectivamente, que no presente trabalho. No trabalho de Rizzon e Miele (1998) foram analisadas 13 amostras de vinagres de vinho branco e 16 amostras de vinagres de vinho tinto.

\section{Acidez volátil}

A acidez do vinagre exerce grande influência na aceitação sensorial do produto, sendo o percentual de ácido acético dos vinagres diretamente proporcional à acidez percebida sensorialmente (GRANANDA et al., 2000; TESFAYE et al., 2002). A legislação brasileira exige um mínimo de 4,00\% de ácido acético em vinagres (BRASIL, 1999), de forma que a difusão comercial e a aceitação dos produtos devem embasarse no conhecimento de suas propriedades sensoriais, as quais contribuem para valorizar e proteger a genuinidade destes produtos (GRANANDA et al., 2000). Das amostras analisadas, apenas os vinagres de vinho tinto (VVT), vinagre de maçã (VMç) e vinagre de vinho branco (VVB) apresentaram valores de acidez volátil em conformidade com a legislação (BRASIL, 1999). Os demais produtos analisados apresentaram valores, para esta determinação, aquém do permitido para classificálos como fermentados acéticos ou vinagres. Ressalta-se que, de acordo com os resultados para acidez volátil expostos na Tabela 1, apenas os VVT, VMç e VVB podem ser classificados, por legislação, como fermentados acéticos ou vinagres. Os vinagres de laranja com mel (VLMl), laranja (VL), tangerina com milho (VTgMi), manga (VMg), arroz (Var), kiwi (VK), cana-de-açúcar (VC), maracujá (VMr), cana-de-açúcar com milho (VCMi) e milho (VMi) apresentaram valores de acidez volátil abaixo dos determinados pela legislação vigente (BRASIL, 1999), sendo os VLMl e VL os que apresentaram menores valores, 2,53 e 2,74\%, respectivamente. É necessário, portanto considerar a tecnologia de produção destes vinagres, uma vez que a composição química do vinagre está relacionada com a qualidade, a tecnologia e a matéria-prima utilizada na sua elaboração (RIZZON; MIELE, 1998).
Em relação aos dados encontrados e explicitados na Tabela 1 é possível observar que não houve diferença significativa ( $\mathrm{p}>0,05)$ entre os vinagres de maracujá (VMr), milho (VMi), cana-de-açúcar (VC) e tangerina (VTg), que apresentaram valores entre 3,75 e 4,01\%. Houve diferença significativa ( $\mathrm{p} \leq 0,05)$ nos valores de acidez volátil do vinagre de vinho tinto (5,00\%) e vinho branco (4,03\%). Rizzon e Miele (1998) encontraram valores médios de acidez volátil de $4,4 \%$ para vinagre de vinho tinto e $4,3 \%$ para vinagre de vinho branco.

\section{pH dos vinagres}

Tanto a acidez quanto os valores de $\mathrm{pH}$ influenciam diretamente as características sensoriais dos vinagres. Em vinagres com acidez em torno de 5\%, esperam-se intervalos de $\mathrm{pH}$ em torno de 2,46 a 3,18, valores dependentes do tipo de vinagre a ser analisado, como vinagres provenientes de destilados, vinagres de vinho, vinagre de maçã, etc. (WHITE, 1971).

Os vinagres de tangerina (VTg) e tangerina com milho (VTgMi) não diferiram significativamente $(\mathrm{p}>0,05)$ entre $\mathrm{si}$, com valores de 3,51 e 3,54, respectivamente, assim como os vinagres de milho (VMi) e cana-de-açúcar com milho (VCMi), com valores entre 3,05 e 3,03, respectivamente.

Quanto ao $\mathrm{pH}$, os vinagres de vinho tinto (VVT, 2,69) e de vinho branco (VVB, 2,88) diferiram significativamente ( $\mathrm{p} \leq 0,05)$ entre si, mas aproximaram-se da média de $\mathrm{pH}$ encontrada no trabalho de Rizzon e Miele (1998), de 2,77 para vinagre de vinho tinto (cerca de $3 \%$ maior que no VVT) e 2,75 para vinagre de vinho branco (aproximadamente $4,5 \%$ menor que no $\mathrm{VVB}$ ).

\section{Cinzas}

O resíduo por incineração ou cinzas é o nome dado ao resíduo obtido por aquecimento de um produto em temperatura próxima a 550 e $570{ }^{\circ} \mathrm{C}$. Geralmente, as cinzas são obtidas por ignição de quantidade conhecida da amostra e representam o conteúdo inorgânico, ou seja, mineral da amostra (INSTITUTO ADOLFO LUTZ, 2005; RIZZON; MIELE, 2001, 2002), com quantidade dependente do processo e da matériaprima utilizados em sua produção (PALMA; CARVALHO; GAVÓGLIO, 2001).

Observou-se uma diferença significativa $(\mathrm{p} \leq 0,05)$ entre os VVT $\left(1,49\right.$ g.L. L $\left.^{-1}\right)$ e VVB $\left(0,72\right.$ g. $\left.L^{-1}\right)$. Comparando-se com os valores encontrados por Rizzon e Miele (1998), os valores de cinzas para o vinagre de vinho tinto $\left(1,46\right.$ g. $\left.\mathrm{L}^{-1}\right)$ aproximam-se dos encontrados no presente trabalho, cerca de $2 \%$ inferiores. Porém há uma evidente diferença no teor de cinzas em relação ao vinagre de vinho branco (1,50 g. $\left.\mathrm{L}^{-1}\right)$ analisado por Rizzon e Mielle (1998), que apresentou valor em torno de 52\% superior ao VVB analisado no presente trabalho.

Vinagres como laranja com mel (VLMl, 5,14 g.L $\left.\mathrm{L}^{-1}\right)$, laranja (VL, 3,65 g. $\mathrm{L}^{-1}$ ), tangerina com milho (VTgMi, 3,46 g.L $\mathrm{L}^{-1}$ ), tangerina (VTg, 2,93 g. $\left.\mathrm{L}^{-1}\right)$, manga (VMg, 2,25 g. $\left.\mathrm{L}^{-1}\right)$ e arroz (Var, 3,75 g.L $\mathrm{L}^{-1}$ ) apresentaram altos teores de cinzas quando comparados aos vinagres convencionais de vinho tinto (VVT) 
e vinho banco (VVB). Sabendo-se que a qualidade do vinagre é dependente da matéria-prima e do seu processo de produção (PALMA; CARVALHO; GAVÓGLIO, 2001), acredita-se que o tipo de matéria-prima utilizada na sua produção (laranja, mel, tangerina, manga, milho e arroz) bem como a filtração dos produtos durante a fabricação podem ter interferido na prevalência de valores elevados para esta determinação.

Não apresentaram diferença significativa $(p>0,05)$ os vinagres de vinho tinto (VVT), maracujá (VMr), maçã (VMç) e kiwi (VK), com valores entre 1,45 e 1,66 g.L-1. Além disso, não houve diferença significativa $(\mathrm{p}>0,05)$ entre os vinagres de milho (VMi), vinho branco (VVB) e cana-de-açúcar (VC), 0,72 a 0,91 g.L $L^{-1}$.

\section{Grau alcoólico real}

Quanto ao grau alcoólico real, todas as amostras realizadas apresentaram valores de $0 \%$ de álcool, de acordo com a tabela de conversão de densidade relativa do destilado a $20{ }^{\circ} \mathrm{C}$ em porcentagem de álcool em peso, não sendo os dados expressos na Tabela 1. Possivelmente, a metodologia para determinação do teor alcoólico não apresentou sensibilidade suficiente para detectar concentrações baixas presentes nos fermentados acéticos, apesar de ser a metodologia indicada por legislação (BRASIL, 2005) para análises de vinagres. Rizzon e Miele (1998) estudaram a composição analítica de vinagres brasileiros de vinho tinto e vinho branco e encontraram valores médios de 0,13 e $0,15 \%$, respectivamente. Segundo White (1971), considera-se eficiente uma conversão de álcool em ácido acético na ordem de $70 \%$, podendo chegar a uma eficiência de 90 a $98 \%$. Assim, o conteúdo alcoólico de um vinagre deve ser pequeno, uma vez que praticamente todo o álcool etílico pré-existente deve converter-se em ácido acético mediante a fermentação acética (ARTILES; ROMERO; TORRE, 1993). A legislação não determina um valor mínimo para conteúdo de álcool em vinagres, apenas determina o valor máximo, de 1,0\% em volume a $20^{\circ} \mathrm{C}$ (BRASIL, 1999).

É importante considerar que é desejável que existam pequenas quantidades de álcool residual no vinagre produzido, pois isso repercutirá favoravelmente no flavor dos vinagres, por formação do buquet, principalmente se houver armazenamento do produto durante alguns meses. Além disso, principalmente em se tratando de armazenamento a longo prazo, o consumo de todo o álcool dos vinagres, favorece o consumo pelas bactérias acéticas de ácido acético, originando produtos de baixa qualidade. Destaca-se também que métodos lentos de produção de vinagres originam vinagres com um conteúdo alcoólico maior, uma vez que não é possível obter-se rendimentos de ácido acético tão elevados a partir do álcool etílico inicial, assim como acontece nos métodos de elaboração de vinagres rápidos (industriais) (ARTIILES, ROMERO; TORRE, 1993).

\section{Açúcares redutores pelo método do ADNS}

Para a determinação dos valores numéricos de açúcares redutores, foi utilizada a equação de regressão $(y=0,3834 . x-0,3561)$, oriunda da curva padrão estabelecida entre a concentração de glicose $\left(\mathrm{mg} \cdot \mathrm{mL}^{-1}\right)$ e a leitura das respectivas absorbâncias a $540 \mathrm{~nm}$, em que $\times$ corresponde à quantidade de açúcar das amostras (g.L-1 $)$ e y à absorbância das amostras $(760 \mathrm{~nm})$ com coeficiente de determinação $\mathrm{R}^{2} 0,9992$.

Houve diferença significativa no teor de açúcares redutores de vinagre de vinho tinto (VVT, 1,57 g. $\mathrm{L}^{-1}$ ) e vinagre de vinho branco (VVB, 1,12 g.L $\mathrm{L}^{-1}$ ). Rizzon e Miele (1998), utilizando método titulométrico para determinação de açúcares redutores, encontraram uma média de 1,88 g. $\mathrm{L}^{-1}$ para vinagres de vinho tinto e 2,04 g. $\mathrm{L}^{-1}$ para vinagres de vinho branco, valores cerca de 20 e $82 \%$ superiores, respectivamente, que os encontrados no presente trabalho. Ressalta-se, porém, que as determinações dos referidos autores apresentaram valores mínimos 0,7 e 0,4 g.L. $\mathrm{L}^{-1}$ (vinagre de vinho tinto e branco, respectivamente) e máximos de 3,1 g.L. $\mathrm{L}^{-1}$ para os dois tipos de vinagres. Mais uma vez concluise que a composição química do vinagre está relacionada com a qualidade, a tecnologia, a fermentação e a matéria-prima utilizada na sua elaboração (RIZZON; MIELE, 1998).

O maior teor de açúcares redutores foi do vinagre de manga (VMg, 8,74 g. $\mathrm{L}^{-1}$ ), seguido dos vinagres de arroz (Var, 6,65 g.L $\left.\mathrm{L}^{-1}\right)$, maçã (VMç, 6,22 g.L $\mathrm{L}^{-1}$ ). Assim, observa-se que a grande quantidade de açúcares redutores encontrados nos vinagres derivados de manga, arroz e maçã, justificam-se pela presença considerável de amido (como fonte de glicose) nestas matérias-primas.

Além disso, a inserção do milho no vinagre de tangerina com milho (VTgMi, 2,53 g. $\left.\mathrm{L}^{-1}\right)$ em comparação ao vinagre de tangerina (VTg, 2,75 g.L $\mathrm{L}^{-1}$ ) não influenciou na quantidade de açúcares redutores das amostras, uma vez não diferiram significativamente $(p>0,05)$ entre si. O mesmo pode-se observar em relação aos vinagres de cana-de-açúcar com milho (VCMi, 1,31 g. $\mathrm{L}^{-1}$ ) e milho (VMi 1,13 g.L. $\mathrm{L}^{-1}$ ). Os vinagres de laranja (VL, 4,12 g. $\mathrm{L}^{-1}$ ) e laranja com mel (VLMl, 4,35 g. $\mathrm{L}^{-1}$ ) também não apresentaram diferença significativa $(\mathrm{p}>0,05)$.

\subsection{Parâmetros nutricionais: polifenóis totais em vinagres}

Os compostos fenólicos são estruturas químicas que apresentam hidroxilas e anéis aromáticos, nas formas simples ou polímeros, que lhes confere poder antioxidante, destacando-se dentre eles os flavonoides, ácidos fenólicos, taninos e tocoferóis, como antioxidantes fenólicos mais comuns de fonte natural. São compostos incluídos na categoria de interruptores de radicais livres, sendo eficientes na prevenção da autoxidação, apresentando ações importantes na redução do risco de desenvolvimento de patologias, como arteriosclerose e câncer (ANGELO; JORGE, 2007; DEGÁSPARI; WASZCZYNSKYJ, 2004; KÄHKÖNEN et al., 1999; YANG et al., 1997).

Para a determinação dos valores numéricos de polifenóis totais foi utilizada a equação de regressão $(y=0,003984 x-0,003347)$ oriunda da curva padrão estabelecida entre a concentração de ácido gálico $\left(\mathrm{mg} \cdot \mathrm{mL}^{-1}\right)$ e a leitura das respectivas absorbâncias a $760 \mathrm{~nm}$, em que $\mathrm{x}$ corresponde à quantidade de polifenóis totais das amostras (mg EAG.100 mL $\mathrm{m}^{-1}$ e y à absorbância das amostras $(760 \mathrm{~nm})$ com coeficiente de determinação $\mathrm{R}^{2}$ 0,9998. 
O conteúdo de polifenóis totais provenientes de vinagre de vinho tinto VVT, 31,86 mg EAG .100 $\mathrm{mL}^{-}$) encontra-se no limite mínimo do detectado por Dávalos, Bartolomé e Gómez-Cordovés (2005), que estudaram as propriedades antioxidantes de sucos comerciais de uva, vinhos e vinagres, e detectaram uma variação entre 30,6 e 86,7 mg EAG. $100 \mathrm{~mL}^{-1}$ para vinagres.

Não houve diferença significativa $(\mathrm{p}>0,05)$ entre os vinagres de laranja (VL, 28,83 mg EAG.100 $\mathrm{mL}^{-1}$ ) e tangerina com milho (VTgMi, 27,61 mg EAG.100 $\mathrm{mL}^{-1}$ ), porém os vinagres de laranja (VL) e laranja com mel (VLMl, 43,27 mg EAG.100 $\mathrm{mL}^{-1}$ ) diferiram significativamente $(\mathrm{p} \leq 0,05)$ entre si. A presença do mel na formulação do vinagre de laranja com mel $\left(43,27 \mathrm{mg}\right.$ EAG.100 $\left.\mathrm{mL}^{-1}\right)$ contribuiu para o aumento do conteúdo de polifenóis totais em relação ao vinagre de laranja (28,83 mg EAG.100 $\left.\mathrm{mL}^{-1}\right)$ em torno de 50\%. É necessário, porém, considerar a origem do mel utilizado, uma vez que as composições dos méis variam grandemente conforme a espécie de abelha produtora, bem como da sua origem floral, geográfica e de safra para safra, envolvendo condições climáticas (PEREIRA; QUEIROZ; FIGUEIRÊDO, 2003; QUEIROZ et al., 2007). Sendo assim, sugere-se a realização de estudos para verificar os teores de polifenóis totais em vinagres de mel, utilizando matéria-prima de diferentes origens e considerando as características particulares do mel mencionadas acima, como espécie de abelha e origem floral.

É válido ressaltar que o VLMl apresentou teor de polifenóis totais $\left(43,27 \mathrm{mg}\right.$ EAG. $\left.100 \mathrm{~mL}^{-1}\right)$ maior que o vinagre de vinho tinto (VVT, 31,86 mg EAG.100 $\mathrm{mL}^{-1}$ ), o qual apresenta como matéria-prima básica a uva. Segundo Dávalos, Bartolomé e Gómez-Cordovés (2005), frutas derivadas Vitis vinifera apresentam maior concentração e variedade de compostos fenólicos. Em estudo realizado por Sun et al. (2002) avaliando a atividade antioxidante de frutas comuns, verificou-se que $o$ teor de compostos fenólicos em uvas vermelhas $\left(182,0 \mathrm{~g} .100 \mathrm{~g}^{-1}\right)$ foi significativamente superior ao encontrado em laranjas $\left(53,6 \mathrm{mg} \cdot 100 \mathrm{~g} \mathrm{~g}^{-1}\right)$. Este dado nos remete à inferência de que o resultado superior de compostos fenólicos encontrado em vinagre de laranja com mel (VLMl) em relação ao vinagre de vinho tinto (VVT) refere-se, possivelmente, à quantidade superior da referida fruta utilizada no processamento do vinagre, em relação ao vinagre de vinho tinto. Além disso, independentemente do produto, a predominância dos compostos fenólicos pode sofrer alterações de acordo com a procedência e o tipo da uva (MAMEDE; PASTORE, 2004). O processo de elaboração do vinagre também deve ser considerado, uma vez que, de acordo com White (1971), o vinagre de vinho é oriundo de um processo duplo de fermentação utilizando como matéria-prima a uva, e o vinagre de vinho de frutas pode ser produzido de frutas que não sejam uvas, ou da mistura de uvas e outras frutas. Vale lembrar mais uma vez que a composição química do vinagre está relacionada com a qualidade, a tecnologia e a matériaprima utilizada na sua elaboração (PALMA; CARVALHO; GAVÓGLIO, 2001; RIZZON; MIELE, 1998).

Houve diferença significativa $(\mathrm{p} \leq 0,05)$ também entre os vinagres de tangerina (VTg) e tangerina com milho
(VTgMi), sendo que o VTgMi apresentou conteúdo de polifenóis totais menor (27,61 mg EAG.100 $\left.\mathrm{mL}^{-1}\right)$ que o VTg $\left(34,36 \mathrm{mg}\right.$ EAG $\left.100 \mathrm{~mL}^{-1}\right)$. Este fato possivelmente está associado à presença do milho na composição dos vinagres, pois o vinagre de milho (VMi) apresentou o menor conteúdo de polifenóis totais dentre as amostras $\left(3,13 \mathrm{mg}\right.$ EAG $\left.100 \mathrm{~mL}^{-1}\right)$.

Com os vinagres de cana-de-açúcar (VC, 9,66 mg EAG.100 mL $\mathrm{mL}^{-1}$ ) e cana-de-açúcar com milho (VCMi, $5,13 \mathrm{mg}$ EAG. $100 \mathrm{~mL}^{-1}$ ), observou-se que, novamente, a adição do milho na composição do vinagre resultou em amostra com conteúdo menor de polifenóis totais. Não houve diferença significativa $(p>0,05)$ entre os vinagres de cana-de-açúcar com milho e vinagre de arroz (Var, 6,54 mg EAG. $100 \mathrm{~mL}^{-1}$ ). Ressaltase que o vinagre de arroz analisado não é oriundo de arroz integral (não polido), e sim de arroz polido, o que diminui o seu valor nutricional e principalmente o conteúdo de polifenóis totais. Nishidai et al. (2000) determinaram o teor de polifenóis totais de extratos (à base de acetato de etila) de diferentes tipos de vinagres e observaram que o valor encontrado para o vinagre produzido a partir de arroz não polido $\left(112 \mathrm{mg} .100 \mathrm{~mL}^{-1} \mathrm{de}\right.$ extrato) foi quase duas vezes superior ao encontrado no vinagre de arroz polido (64 mg $100 . \mathrm{mL}^{-1}$ de extrato). Vale ressaltar que Nishidai et al. (2000) trabalharam com extratos de vinagres produzidos à base de acetato de etila. No presente trabalho, trabalhou-se com as amostras de vinagre sem nenhum tipo de extração com álcool e/ou concentração das amostras, uma vez que se pretendeu conhecer a composição química das amostras da forma como elas são comercializadas no mercado.

Não houve diferença significativa $(\mathrm{p}>0,05)$ entre os vinagres de kiwi $(\mathrm{VK})$ e manga $(\mathrm{VMg})$, com teores médios de 17,61 e 16,80 mg EAG.100 mL ${ }^{-1}$, respectivamente. Além disso, os vinagres de maracujá (VMr), cana-de-açúcar (VC) e vinagre de vinho branco (VVB) também não diferiram entre si $(\mathrm{p}>0,05)$, variando em torno de 9,53 a 9,79 mg EAG.100 mL $\mathrm{mL}^{-1}$.

Quanto ao vinagre de vinho tinto (VVT), este apresentou teor de polifenóis totais de $31,86 \mathrm{mg}$ EAG. $100 \mathrm{~mL}^{-1}$, valor próximo (cerca de 6\% aquém) ao encontrado em extrato de vinagre de vinho tinto por Nishidai et al. (2000) (34,00 mg.100 mL de extrato), semelhante também aos encontrados por Dávalos, Bartolomé e Gómez-Cordovés (2005), que detectaram uma variação de 30,6 a $86,7 \mathrm{mg}$ EAG. $100 \mathrm{~mL}^{-1}$.

Os teores de polifenóis totais dos vinagres de laranja com mel (VLMl, 43,27 mg EAG.100 mL ${ }^{-1}$ ) e tangerina (VTg, $34,36 \mathrm{mg}$ EAG. $100 \mathrm{~mL}^{-1}$ ) superaram o teor do VVT. O vinagre de vinho branco apresentou somente 9,53 mg EAG. $100 \mathrm{~mL}^{-1} \mathrm{de}$ polifenóis totais. Bravo (1998) detectou em vinagres de vinho tinto valores entre 100 e $400 \mathrm{mg} .100 \mathrm{~mL}^{-1}$ de polifenóis totais e, em vinho branco, de 20 a $30 \mathrm{mg} \cdot 100 \mathrm{~mL}^{-1}$. A diferença na quantidade de compostos fenólicos dos vinhos tintos e brancos não se deve apenas à presença de antocianinas, mas também aos processos de fabricação para obtenção do vinho. Em alguns tipos de vinho, as uvas são esmagadas com engaço, casca e semente, gerando maior quantidade de compostos fenólicos (MAMEDE; PASTORE, 2004). 
Existe uma literatura abundante abordando a ação antioxidante individual de compostos fenólicos. Porém, pouco se sabe ainda sobre a influência da interação de vários compostos fenólicos sobre a capacidade antioxidante (PINELO et al., 2004). Além disso, é necessário realizar a quantificação da capacidade antioxidante das amostras, uma vez que somente o teor de polifenóis totais não a determina.

É possível considerar então que, além do conteúdo de polifenóis totais, outros fatores podem interferir na capacidade antioxidante de vinagres, como por exemplo, a própria constituição de polifenóis totais. A maior parte da literatura que aborda os polifenóis totais como compostos antioxidantes analisa a ação individual destes compostos. Porém, pouco se conhece sobre a influência da interação de vários compostos fenólicos sobre a capacidade antioxidante, podendo estes apresentar, inclusive, efeito sinérgico negativo sobre a capacidade antioxidante da amostra (PINELO et al., 2004). Além disso, Dávalos, Bartolomé e Gómez-Codrovés (2005) afirmaram que, em produtos derivados de uva (sucos, vinhos e vinagres), a safra de produção desta, a variedade de uva, técnicas de produção de mostos e circunstâncias de envelhecimento de produtos influenciam no conteúdo de polifenóis totais. Sendo assim, sugere-se, além da quantificação dos teores de polifenóis totais, a identificação e quantificação destes, individualmente nas amostras, bem como da sua capacidade antioxidante, com o intuito de se conhecer as possíveis influências destes compostos fenólicos identificados sobre a capacidade antioxidante dos vinagres.

\section{Conclusões}

Em relação aos parâmetros de identidade e qualidade e características nutricionais dos vinagres, houve heterogeneidade entre as amostras.

Os vinagres de laranja com mel, laranja, tangerina com milho, manga, arroz, kiwi, cana-de-açúcar, maracujá, cana-deaçúcar com milho e milho apresentaram valores de acidez volátil abaixo dos determinados pela legislação vigente e especificado na embalagem.

Houve pouca sensibilidade da metodologia sugerida por legislação para determinação de baixas concentrações de teor alcoólico nas amostras.

Os vinagres de laranja com mel, laranja, tangerina com milho, tangerina, manga e arroz apresentaram teores de cinzas superiores aos vinagres convencionais de vinho tinto e vinho branco. Estes mesmos vinagres apresentaram também teores de extrato seco superiores comparados aos de vinho tinto e branco.

O vinagre de laranja com mel apresentou teor de polifenóis totais maior que o vinagre de vinho tinto. A adição do milho, nos vinagres de tangerina com milho e cana-de-açúcar com milho, influenciou na diminuição do teor de polifenóis totais destas amostras em relação aos vinagres de tangerina e cana-de-açúcar, respectivamente.

\section{Referências bibliográficas}

ANGELO, P. M.; JORGE, N. Compostos fenólicos em alimentos: uma breve revisão. Revista do Instituto Adolfo Lutz, v. 66, n. 1, p. 1-9, 2007.

AQUARONE, E.; ZANCANARO Jr., O. Vinagres. In: AQUARONE, E.; LIMA, U. A.; BORZANI, W. (Eds). Alimentos e bebidas produzidas por fermentação. São Paulo: Edgard Blücher, 1990. p. 104-122.

ARTILES, A. A.; ROMERO, C. D.; TORRE, A. H. Caracterization fisicoquimica de diferentes tipos de vinagres: determination de algunos parametros de naturaleza volátil. Alimentaria, v. 11, p. 105-107, 1993.

BORTOLINI, F.; SANT'ANNA, E. S.; TORRES, R. C. Comportamento das fermentações alcoólica e acética de sucos de kiwi (Actinida deliciosa): composição dos mostos e métodos de fermentação acética. Ciência e Tecnologia de Alimentos, v. 21, n. 2, p. 236-243, 2001.

BRASIL. Ministério da Agricultura, Pecuária e Abastecimento. Instrução Normativa n. 24, de 08 de setembro de 2005. Aprova o manual operacional de bebidas e vinagres. Diário Oficial da União, Poder Executivo, Brasília, DF, 20 de setembro de 2005, Seção 1, p. 11.

BRASIL. Ministério da Agricultura, Pecuária e Abastecimento. Instrução Normativa n. 36, de 14 de outubro de 1999. Aprova o regulamento técnico para fixação dos padrões de identidade e qualidade para fermentados acéticos. Diário Oficial da União, Poder Executivo, Brasília, DF, 15 de outubro de 1999, Seção 1, p. 76.

BRAVO, L. Poliphenols: chemistry, dietary sources, metabolism, and nutritional significance. Revista de Nutrição, v. 56, n. 11, p. 317-333, 1998.

DÁVAlOS, A.; BARTOLOMÉ, B.; GÓMEZ-CORDOVÉS, C. Antioxidant properties of commercial grape juices ant vinegars. Food Chemistry, v. 93, n. 2, p. 325-330, 2005.

DEGÁSPARI, C. H.; WASZCZYNSKYJ, N. Propriedades antioxidantes de compostos fenólicos. Visão Acadêmica, v. 5, n. 1, p. 33-40, 2004.

EBIHARA, K.; NAKAJIMA, A. Effect of acetic acid and vinegar on blood glucose and insulin responses to orally administered sucrose and starch. Agricultural and Biological Chemistry, v. 52, n. 5, p. 1311-1312, 1988.

ENTANI, E. et al. Antibacterial action of vinegar against food-borne pathogenic bacteria including Escherichia coli O157:H7. Journal of Food Protection, v. 61, n. 8, p. 953-959, 1998.

EVANGELISTA, J. Microrganismos e enzimas de utilidade na indústria de alimentos. In:__. Tecnologia de alimentos. 2 ed. São Paulo: Atheneu, 2001. p. 245-281.

GRANANDA, G. G. et al. Vinagres de folhas de videira: aspectos sensoriais. Boletim Centro de Pesquisa de Processamento de Alimentos, v. 18, n. 1, p. 51-56, 2000.

HADFIELD, L. C.; BEARD, L. P.; LEONARD-GREEN, T. K. Calcium content of soup stocks with added vinegar. Journal of the American Dietetic Association, v. 89, n. 12, p. 1810-1811, 1989.

INSTITUTO ADOLFO LUTZ - IAL. Métodos físico-químicos para análise de alimentos. 4 ed. Brasília: Ministério da Saúde, 2005. 1018 p. (Série A, Normas e manuais técnicos).

JOHNSTON, C. S.; BULLER, A. J. Vinegar and peanut products as complementary foods to reduce postprandial glycemia. Journal of the American Dietetic Association, v. 105, n. 12, p. 1939-1942, 2005. 
KÄHKÖNEN, M. P. et al. Antioxidant activity of plant extracts containing phenolic compounds. Journal of Agricultural and Food Chemistry, v. 47, n. 10, p. 3954-3962, 1999.

KUSKOSKI, E. M. et al. Frutos tropicais silvestres e polpas de frutas congeladas: atividade antioxidante, polifenóis e antocianinas. Ciência Rural, v. 36, n. 4, p. 1283-1287, 2006.

MALDONADO, O.; ROLZ, C.; CABRERA, S. S. Wine and vinegar production from tropical fruits. Journal of Food Science, v. 40, n. 2, p. 262-265, 1975.

MAMEDE, M. E. O.; PASTORE, G. M. Compostos fenólicos do vinho: estrutura e ação antioxidante. Boletim Centro de Pesquisa de Processamento de Alimentos, v. 22, n. 2, p. 233-252, 2004.

MILLER, G. L. Use of dinitrosalicylic acid reagent for determination of reducin sugar. Analytical Chemistry, v. 31, n. 3, p. 426-428, 1959.

NISHIDAI, S. et al. Kurosu, a traditional vinegar produced from unpolished rice, suppresses lipid peroxidation in vitro and in mouse skin. Bioscience, Biotechnology and Biochemical, v. 64, n. 9, p. 1909-1914, 2000.

OLIVEIRA, J. A. P. et al. Produção de vinagre e álcool a partir de frutos tropicais excedentes de safra. Boletim Centro de Pesquisa de Processamento de Alimentos, v. 5, n. 1, p. 33-40, 1987.

PALMA, M. S. A.; CARVALHO, L. F. C. P.; GAVÓGLIO, L. C. Vinagres. In: AQUARONE, E. et al. Biotecnologia Industrial. São Paulo: Edgard Blücher, 2001. p. 183-208.

PEREIRA, E. A.; QUEIROZ, A. J. M.; FIGUEIRÊDO, R. M. F. Comportamento reológico de mel da abelha uruçu (Melipona scutellaris, L.). Revista de Ciências Exatas e Naturais, v. 5, n. 2, p. 179-186, 2003.

PINELO, M. et al. Interacion among phenols in food fortification: negative synergism on antioxidant capacity. Journal of Agricultural and Food Chemistry, v. 52, p. 1177-1180, 2004.

QUEIROZ, A. J. M. et al. Comportamento reológico de méis de florada silvestre. Revista Brasileira de Engenharia Agrícola e Ambiental, v. 11, n. 2, p. 190-194, 2007.
REYNERTSON, K. A. et al. Bioactive depsides and anthocyanins from jaboticaba (Myrciaria cauliflora). Journal of Natural Products, v. 69, n. 8, p. 1228-1230, 2006.

RIZZON, L. A.; MIELE, A. Avaliação da Cv. Cabernet Franc para elaboração de vinho tinto. Ciência e Tecnologia de Alimentos, v. 21, n. 2, p. 249-255, 2001.

RIZZON, L. A.; MIELE, A. Avaliação da Cv. Cabernet Sauvigon para elaboração de vinho tinto. Ciência e Tecnologia de Alimentos, v. 22, n. 2, p. 192-198, 2002.

RIZZON, L. A.; MIELE, A. Características analíticas de vinagres comerciais de vinhos brasileiros. Brazilian Journal of Food Technology, v. 1, n. 1.2, p. 25-31, 1998.

STATISTICAL ANALYSIS SYSTEM - SAS. System for Windows: realease 6.12. Cary, 1996.

SEGUN, I. Y.; KARAPINAR, M. Effectiveness of lemon juice, vinegar and their mixture in the elimination of Salmonella typhimurium on carrots (Daucus carota L.). International Journal of Foods Microbiology, v. 96, p. 301-305, 2004.

SOARES, S. E. Ácidos fenólicos como antioxidantes. Revista de Nutrição, v. 15, n. 1, p. 71-81, 2002.

SOUSA, C. M. M. et al. Fenóis totais e atividade antioxidante de cinco plantas medicinais. Química Nova, v. 30, n. 2, p. 351-355, 2007.

SUN, J. et al. Antioxidant and antiproliferative activities of common fruits. Journal of Agricultural and Food Chemistry, v. 50, p. 7449-7454, 2002.

TESFAYE, W. et al. Wine vinegar: technology, authenticity and quality evaluation. Trends in Food Science and Technology, v. 13, p. 12-21, 2002.

WHITE, J. Vinegar quality: legal and commercial standards. Process Biochemistry, p. 21-25, 1971

XU, Q.; TAO, W.; AO, Z. Antioxidant activity of vinegar melanoidins. Food Chemistry, v. 102, n. 3, p. 841-849, 2007.

YANG, J. et al. Varietal differences in phenolic content and antioxidant and antioproliferative acitivties of onions. Journal of Agricultural and Food Chemistry, v. 52, p. 6787-6793, 2004. 